\title{
Finite-time control and synchronization for memristor-based chaotic system via impulsive adaptive strategy
}

\author{
Wei Xiong ${ }^{1}$ and Junjian Huang ${ }^{2 *}$
}

"Correspondence:

jjhuangcqe@163.com

${ }^{2}$ Department of Computer Science,

Chongqing University of Education,

Chongqing, 400067, P.R. China

Full list of author information is

available at the end of the article

\begin{abstract}
This paper investigates the stabilizing and synchronization problems of a memristor-based Chua chaotic system in a finite time. A lemma concerning the finite-time stability for an impulsive system is proposed by extending the finite-time stability theory. Then some finite-time stabilizing and synchronization criterion are presented which guarantee the finite-time stabilization and synchronization for the model considered. Finally, the efficiency of the control scheme is further demonstrated by the simulation examples.
\end{abstract}

Keywords: finite time; control and synchronization; memristor; impulsive adaptive strategy

\section{Introduction}

In 1971, the memristor which is considered to be the missing fourth passive circuit element was postulated [1]. However, this important postulation has not caused attention in almost 40 years. Until in 2008, Hewlett-Packard Labs announced the development of a memristor based on nanotechnology [2]. As we know, the memristor takes its place along with the other three existing elements: the resistor, the capacitor, and the inductor. Increasing focus was put on the memristor for its potential applications in programmable logic, signal processing, neural networks, and so on [3].

Moreover, as the novel element, the circuit based on the memristor shares many interesting phenomenon. Recently, the research memristor chaotic circuits have become a focal topic [4-10]. In [4], the author presented a novel fourth-order memristor-based Chua oscillator by replacing Chua's diode with an active two-terminal circuit. The stabilization problem of a memristor-based chaotic system was investigated in [10]. As the most important phenomenon, the synchronization was also discussed [9]. In [9], the adaptive synchronization problem of memristor-based Chua circuits was investigated.

As time goes on, more and more researchers began to realize the important role of the synchronization time. To attain a high convergence speed, many effective methods have been introduced and finite-time control is one of them. Finite-time synchronization means the optimality in convergence time. Much research work has been done on chaos synchronization based on finite time (see for instance [11-22] and the references

(c) 2016 Xiong and Huang. This article is distributed under the terms of the Creative Commons Attribution 4.0 International License (http://creativecommons.org/licenses/by/4.0/), which permits unrestricted use, distribution, and reproduction in any medium, provided you give appropriate credit to the original author(s) and the source, provide a link to the Creative Commons license, and indicate if changes were made. 
Figure 1 Chua's memristor-based chaotic circuits with a flux-controlled memristor.

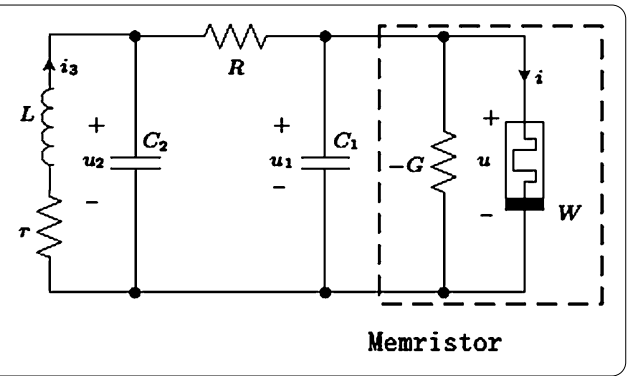

therein). However, the finite-time synchronization problem has not been fully investigated in the literature, and it still remains open. Motivated by the above discussion, we investigate the finite-time synchronization problem for a memristor-based Chua circuit. Based on the finite-time stability theory, a novel lemma which guarantees the impulsive system is finite-time stable is presented. Then the impulsive [16-18] adaptive control law is proposed to realize finite-time synchronization of the model considered. Numerical simulations demonstrate the effectiveness and correctness of this results.

The paper is organized as follows. Some preliminaries are presented in the next section. Section 3 proposes the main results of this paper. In Section 4, the numerical simulations are presented, which is followed by the conclusion in Section 5 .

\section{Preliminaries}

In [6], the author proposed a novel nonlinear circuits, with a flux-controlled memristor which replaces the Chua diode. Figure 1 shows a memristor-based Chua oscillator with a flux-controlled memristor.

Applying a Kirchhoff voltage, the current law, and the volt-ampère relationship of the components, the state equation of a Chua memristor-based chaotic system can be described as follows:

$$
\left\{\begin{array}{l}
\dot{u}_{1}=\frac{1}{C_{1}}\left(u_{2}-u_{1}+G u_{1}-W(\phi) u_{1}\right), \\
\dot{u}_{2}=\frac{1}{C_{2}}\left(u_{1}-u_{2}\right)+R i_{3}, \\
\dot{i}_{3}=-\frac{1}{L} u_{2}+\frac{r}{L} i_{3} \\
\dot{\phi}=u_{1}
\end{array}\right.
$$

For convenience, letting $x_{1}=u_{1}, x_{2}=u_{2}, x_{3}=i_{3}, x_{4}=\phi, \alpha=1 / C_{1}, \beta=1 / L_{1}, \gamma=r / L, \xi=G$, $C_{2}=1$, and $R=1$, then the model can be rewritten as the following equation:

$$
\left\{\begin{array}{l}
\dot{x}_{1}=\alpha\left(x_{2}-x_{1}+\xi x_{1}-W\left(x_{4}\right) x_{1}\right) \\
\dot{x}_{2}=x_{1}-x_{2}+x_{3} \\
\dot{x}_{3}=-\beta x_{2}+\gamma x_{3} \\
\dot{x}_{4}=x_{1} .
\end{array}\right.
$$

If we set $\alpha=10, \beta=100 / 7, \gamma=0.1, \xi=9 / 7, a=1 / 7$ and $b=2 / 7$, and the initial values are $\left(10^{-10}, 0,0,-0.515\right)$, then system (2) is a chaotic system and the chaotic attractor is shown in Figure 2.

In order to compute simply, letting $x=\left[x_{1}, x_{2}, x_{3}, x_{4}\right]^{T}$, then system (2) can be described as follows:

$$
\dot{x}=A x+\phi(x),
$$


Figure 2 The chaotic attractor of the memristor-based Chua circuit.

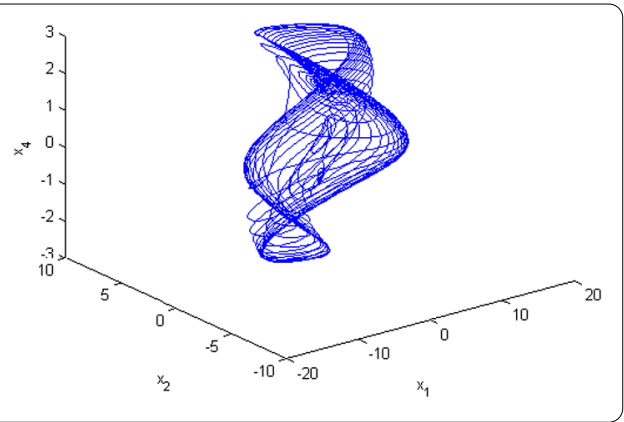

where

$$
A=\left[\begin{array}{cccc}
\alpha(\xi-1) & \alpha & 0 & 0 \\
1 & -1 & 1 & 0 \\
0 & -\beta & \gamma & 0 \\
1 & 0 & 0 & 0
\end{array}\right], \quad \phi(x)=\left[\begin{array}{c}
-\alpha W\left(x_{4}\right) x_{1} \\
0 \\
0 \\
0
\end{array}\right]
$$

and $W\left(x_{4}\right)=a+3 b x_{4}^{2}, a, b, \gamma, \xi, \alpha$, and $\beta$ are positive constants.

Similar to [7], the nonlinear functions $q(\phi), W(\phi)$ are given by

$$
\begin{aligned}
& q(\phi)=a \phi+b \phi^{3}, \\
& W(\phi)=\frac{d q(\phi)}{d \phi}=a+3 b \phi^{2} .
\end{aligned}
$$

Throughout this paper, the following assumption and lemma are necessary for our main results.

Assumption 1 System (2) is a chaotic system, namely, the state is bound, we assume that the following assumptions hold:

$$
\left|x_{1}\right| \leq M_{1}, \quad\left|x_{4}\right| \leq M_{2}
$$

where $M_{1}, M_{2}$ are real constants.

Lemma 1 Suppose the function is continuous and non-negative when $t \in[0, \infty)$ and satisfies the following conditions:

$$
\left\{\begin{array}{l}
\dot{V}(t) \leq-\rho V^{\eta}(t), \quad t \neq t_{k} \\
V\left(t_{k}^{+}\right) \leq \delta V\left(t_{k}\right), \quad k=1,2, \ldots
\end{array}\right.
$$

where $\rho>0,0<\eta<1,0<\delta<1, k=\{1,2, \ldots, m\}$, is a finite natural number set and $m$ is a positive integer, then the following inequality holds:

$$
V^{1-\eta}(t) \leq V^{1-\eta}\left(t_{0}\right)-\rho(1-\eta)\left(t-t_{0}\right), \quad t_{0} \leq t \leq T,
$$

where $T$ is a constant which represents the setting time. 
Proof Without loss of generality, let $t_{0}=0$. In order to prove (7) holds, the following function $H(t)$ is constructed:

$$
H(t)=V^{1-\eta}(t)-V^{1-\eta}(0)+\rho(1-\eta) t .
$$

Clearly, if the function $H(t)$ satisfies $H(t) \leq 0$, then the equality (7) holds.

One can easily observe that

$$
H(0)=V^{1-\eta}(0)-V^{1-\eta}(0) \leq 0 .
$$

Next, we will prove that $H(t) \leq 0$ holds for $t \in\left[t_{0}, t_{1}\right]$. Otherwise, there exists $t^{*}$ such that

$$
\begin{aligned}
& H(t)>0, \quad t \in\left(t^{*}, t_{1}\right), \quad H(t)<0, \quad t \in\left(t_{0}, t^{*}\right), \\
& H\left(t^{*}\right)=0, \quad \dot{H}\left(t^{*}\right)>0 .
\end{aligned}
$$

Combing (10) with (11), one has

$$
\begin{aligned}
\dot{H}\left(t^{*}\right) & =(1-\eta) V^{-\eta}\left(t^{*}\right) \dot{V}\left(t^{*}\right)+\rho(1-\eta) \\
& =(1-\eta) V^{-\eta}\left(t^{*}\right)\left(-\rho V^{-\eta}\left(t^{*}\right)\right)+\rho(1-\eta) \\
& =0
\end{aligned}
$$

which contradicts (11). Namely, $H(t) \leq 0$ holds for $t \in\left[t_{0}, t_{1}\right)$.

When $t=t_{1}$, we get

$$
\begin{aligned}
H\left(t_{1}\right)-H\left(t_{1}^{-}\right) & =H\left(t_{1}^{+}\right)-H\left(t_{1}^{-}\right) \\
& =V^{1-\eta}\left(t_{1}^{+}\right)-V^{1-\eta}\left(t_{1}^{-}\right)=\left(\beta^{1-\eta}-1\right) V^{1-\eta}\left(t_{1}^{-}\right) \\
& \leq 0 .
\end{aligned}
$$

It yields

$$
H\left(t_{1}\right) \leq H\left(t_{1}^{-}\right) \leq 0 .
$$

Then we suppose that $H(t) \leq 0$ holds for $t \in\left[t_{k-1}, t_{k}\right]$. For $t \in\left[t_{k}, t_{k+1}\right]$, we have

$$
\begin{aligned}
H\left(t_{k+1}\right) & =V^{1-\eta}\left(t_{k+1}\right)-V^{1-\eta}(0)+\alpha(1-\eta) t_{k+1} \\
& =\beta^{1-\eta} V^{1-\eta}\left(t_{k+1}^{-}\right)-V^{1-\eta}(0) \alpha(1-\eta) t_{k+1}^{-} \\
& =\left[\beta^{1-\eta}-1\right] V^{1-\eta}\left(t_{k+1}^{-}\right)-H\left(t_{k+1}^{-}\right) \\
& \leq\left[\beta^{1-\eta}-1\right] V^{1-\eta}\left(t_{k+1}^{-}\right)-H\left(t_{k}^{+}\right) \\
& \leq 0
\end{aligned}
$$

From (8)-(15), we know that $H(t) \leq 0$ holds. This completes the proof. 


\section{Main results}

In this section, the finite-time control and synchronization problems via an impulsive adaptive strategy are investigated, respectively. Taking the impulsive adaptive strategy into account in (3), one obtains

$$
\begin{cases}\dot{x}(t)=A x(t)+\phi(x(t))+u(t), & t \neq t_{k}, \\ \Delta x\left(t_{k}\right)=I_{k}\left(x\left(t_{k}\right)\right)=-B x\left(t_{k}\right), & t=t_{k}, k \in \ell\end{cases}
$$

where $\Delta x\left(t_{k}\right)=x\left(t_{k}^{+}\right)-x\left(t_{k}^{-}\right), x\left(t_{k}^{+}\right)=\lim _{t \rightarrow t_{k}^{+}} x(t), x\left(t_{k}^{-}\right)=\lim _{t \rightarrow t_{k}^{-}} x(t), \ell=\left\{1,2, \ldots, n, n_{1}, \ldots\right.$, $\left.n_{k}\right\}$, is a finite natural number set. For simplicity, it is assumed that $x\left(t_{k}^{-}\right)=x\left(t_{k}\right)$, which means that $x\left(t_{k}\right)$ is left continuous. Letting $u(t)=-k_{1} x(t)-k_{2} \operatorname{sign}(x(t))|x(t)|^{\gamma}$, we have the following theorem.

Theorem 1 Suppose Assumption 1 holds. There exists a positive constant $\gamma$ satisfying $0<$ $\gamma<1$ such that the memristor-based chaotic system is finite-time stable if the following conditions hold:

(i) $q=\lambda_{\max }\left[A^{T}+A-\left(2 k_{1}+1\right) I\right]<0$;

(ii) $d=\lambda_{\max }(I+B)^{T}(I+B)<1$.

Proof Construct the following Lyapunov candidate function:

$$
V(t, x)=x^{T} x
$$

Calculating the derivative along the trajectory of (16) we have

$$
\begin{aligned}
D^{+} V(t, x) & =\dot{x}^{T} x+x^{T} \dot{x} \\
& =(A x+\phi(x)+u(t))^{T} x+x^{T}(A x+\phi(x)+u(t)) \\
& =x^{T}\left(A+A^{T}-2 k_{1} I\right) x+2 x^{T} \phi(t, x)-2 k_{2} x^{T} \operatorname{sign}(x(t))|x(t)|^{\gamma} .
\end{aligned}
$$

From Assumption 1, one has

$$
\begin{aligned}
2 x^{T} \phi(x) & \leq x^{T} x+\phi^{T}(x) \phi(x) \\
& \leq x^{T} x+\left[\left(a+b x_{4}^{2}\right) x_{1}\right]^{2} \\
& \leq x^{T} x+x_{1}^{2}\left[a+b M_{4}^{2}\right]^{2} \leq(I+J) x^{T} x,
\end{aligned}
$$

where $J=\left[\left[a+b M_{4}^{2}\right]^{2}, 0,0,0\right]^{T}$.

From (18) and (19), one obtains

$$
\begin{aligned}
D^{+} V(t, x) & \leq x^{T}\left[A^{T}+A-\left(2 k_{1}+1\right) I\right] x-2 k_{2} x^{T} \operatorname{sign}(x(t))|x(t)|^{\gamma} \\
& \leq q V(t)-2 k_{2} \sum_{i=1}^{4}\left|x_{i}\right|^{\gamma+1},
\end{aligned}
$$

where $q=\lambda_{\max }\left[A^{T}+A-\left(2 k_{1}+1\right) I\right]$. From the fact that $0<\gamma<1$, one obtains

$$
\left(\sum_{i=1}^{4}\left|x_{i}\right|^{\gamma+1}\right)^{\frac{1}{\gamma+1}} \geq\left(\sum_{i=1}^{4}\left|x_{i}\right|^{2}\right)^{\frac{1}{2}}
$$


Also

$$
\sum_{i=1}^{n}\left|x_{i}\right|^{\gamma+1} \geq\left(\sum_{i=1}^{n}\left|x_{i}\right|^{2}\right)^{\frac{\gamma+1}{2}}=\left(x^{T} x\right)^{\frac{\gamma+1}{2}} .
$$

Then, from condition (1) of Theorem 1 , we have for $t \neq t_{k}$

$$
D^{+} V(t, x) \leq-2 k_{2} V(x)^{\frac{\gamma+1}{2}}
$$

When $t=t_{k}$, one obtains

$$
\begin{aligned}
V\left(t_{k}^{+}\right) & =(x+B x)^{T}(x+B x) \\
& =x\left(I+B^{T}\right)(I+B) x \\
& \leq d V\left(t_{k}\right) .
\end{aligned}
$$

Through condition in Theorem 1 and Lemma 1, we can easily see that the system (16) is finite-time stable, which complete the proof.

Next, we investigate the problem of finite-synchronization for a memristor-based chaotic system. Based on the drive-response synchronization concept, letting system (3) be the drive system, the response system with control input $u$ is as follows:

$$
\dot{y}=A y+\phi(y)+u \text {. }
$$

Taking the impulsive adaptive effects into account, the response system (25) is as follows:

$$
\left\{\begin{array}{l}
\dot{y}=A y+\phi(y)+u(t), \quad t \neq t_{k}, \\
\Delta y=I_{k}(y)=-B e, \quad t=t_{k}, \\
y\left(t_{0}^{+}\right)=y_{0}, \quad t_{0} \geq 0,
\end{array}\right.
$$

where $e=[x-y]^{T}=\left[x_{1}-y_{1}, x_{2}-y_{2}, x_{3}-y_{3}, x_{4}-y_{4}\right]^{T}, t_{k}$ are the impulsive instants which satisfy $t_{1}<t_{2}<\cdots<t_{k-1}<t_{k}$ and $\lim _{k \rightarrow \infty} t_{k}=\infty$. Letting

$$
u=-k_{1} e(t)-k_{2} \operatorname{sign}(e(t))|e(t)|^{\gamma}
$$

where the constants $k_{1}, k_{2}$ are the control strength coefficients to be designed, the real number $\gamma$ satisfies $0 \leq \gamma<1$.

Combing (3) with (26), the error system can be described as follows:

$$
\left\{\begin{array}{l}
\dot{e}=A e+\phi(e)+u(t), \quad t \neq t_{k} \\
\Delta e=B e, \quad t=t_{k}, k=1,2, \ldots
\end{array}\right.
$$

where $\phi(e)=\left[\alpha W\left(y_{4}\right) y_{1}-\alpha W\left(x_{4}\right) x_{1}, 0,0,0\right]^{T}$. Hence, we have the following theorem. 
Theorem 2 Suppose Assumption 1 holds. There exists a positive constant $\gamma$ satisfying $0<$ $\gamma<1$ such that the memristor-based Chua systems (3) and (26) can be synchronized under the impulsive adaptive strategy if the following conditions hold:

(i) $A^{T}+A-2 k_{1} I-2 a b M_{1} M_{2} I<0$;

(ii) $d=\lambda_{\max }(I+B)^{T}(I+B)<1$.

Proof Construct the following Lyapunov candidate function

$$
V(e)=e^{T} e .
$$

For $t \in\left[t_{k}, t_{k+1}\right)$, the derivative of $V(t)$ along the trajectory of (28) is

$$
D^{+} V(e)=2 e^{T} \dot{e}=2 e^{T}\left(A-k_{1} I\right) e(t)+2 e^{T} \phi(e)-2 k_{2} e^{T} \operatorname{sign}(e(t))|e(t)|^{\alpha} .
$$

From Assumption 1, we have

$$
\begin{aligned}
2 e^{T} \phi(e) & =2 \alpha\left[\left(a+b y_{4}^{2}\right) y_{1}-\left(a+b x_{4}^{2}\right) x_{1}\right] e_{1} \\
& =-2 \alpha e_{1}^{2}+2 a b\left(y_{4}^{2} y_{1}-y_{4}^{2} x_{1}+y_{4}^{2} x_{1}-x_{4}^{2} x_{1}\right) \\
& \leq 2 a b M_{1} M_{2} e^{T} e .
\end{aligned}
$$

Combing (30) with (31), we have

$$
D^{+} V(e) \leq e^{T}\left(A^{T}+A-2 k_{1} I-2 a b M_{1} M_{2} I\right) e-2 k_{2} \sum_{i=1}^{4}\left|e_{i}\right|^{\gamma+1}
$$

From the fact that $0<\gamma<1$, one obtains

$$
\left(\sum_{i=1}^{4}\left|e_{i}\right|^{\gamma+1}\right)^{\frac{1}{\gamma+1}} \geq\left(\sum_{i=1}^{4}\left|e_{i}\right|^{2}\right)^{\frac{1}{2}}
$$

Also

$$
\sum_{i=1}^{n}\left|e_{i}\right|^{\gamma+1} \geq\left(\sum_{i=1}^{n}\left|e_{i}\right|^{2}\right)^{\frac{\gamma+1}{2}}=\left(e^{T} e\right)^{\frac{\gamma+1}{2}}
$$

Thus, based on the condition (i) in Theorem 2 , we have for $t \neq t_{k}$

$$
D^{+} V(e) \leq-2 k_{2} V(e)^{\frac{\gamma+1}{2}} .
$$

When $t=t_{k}$, one obtains

$$
\begin{aligned}
V\left(t_{k}^{+}\right) & =(e+B e)^{T}(e+B e) \\
& =e\left(I+B^{T}\right)(I+B) e \\
& \leq d V\left(t_{k}\right) .
\end{aligned}
$$


Figure 3 Memristor-based chaotic system under an adaptive impulsive strategy.
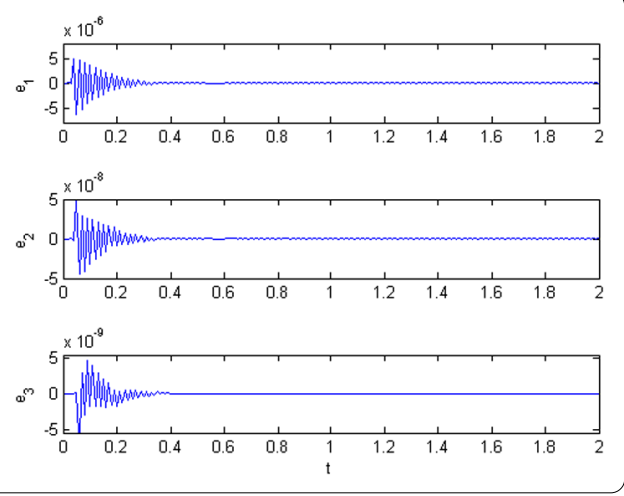

Through Theorem 2 and Lemma 1, we can easily see that the system (28) is finite-time stable. This implies that the response system (26) is synchronized with the drive system (3) in a finite time.

\section{Simulation results}

The numerical simulations are carried out using the fourth-order Runge-Kutta method. The initial states of the drive and response systems are $\left(10^{-10}, 0,0,0\right)$ and $(0,0,0,0)$. The parameters of the drive systems are $\alpha=10, \beta=100 / 7, \gamma=0.1, \xi=9 / 7, a=1 / 7$, and $b=$ $2 / 7$. Solving the inequality in Theorem 2 , and choosing $B=\operatorname{diag}(-0.9,-0.9,-0.9,-0.9)$, $k_{1}=0.02, k_{2}=0.01, \gamma=0.3$, the response system synchronizes with the drive system as shown in Figure 3. It is easily shown that the state response curve of the error system is stable.

\section{Conclusion}

In this paper, the finite-time control and synchronization problems of memristor-based chaotic systems have been investigated. Some novel impulsive adaptive control laws which guarantee the memristor-based Chua circuits is stabilized and synchronized in finite time have been proposed. Moreover, simulation results were given to verify the effectiveness and feasibility of the method. Our future research topics mainly consider the time delay effects on the finite-time stability of the memristor-based nonlinear system.

Competing interests

The authors declare that they have no competing interests.

Authors' contributions

All authors drafted the manuscript, and they read and approved the submitted version.

\section{Author details}

${ }^{1}$ College of Computer, Chongqing College of Electronic Engineering, Chongqing, 401331, P.R. China. ${ }^{2}$ Department of Computer Science, Chongqing University of Education, Chongqing, 400067, P.R. China.

\section{Acknowledgements}

The work described in this paper was partially supported by the National Natural Science Foundation of China (Grant No. 61403050) and the Scientific and Technological Research Program of Chongqing Municipal Education Commission (KJ1501412, KJ1501409, KJ1501301), and the Foundation of CQUE (KY201519B, KY201520B).

Received: 8 November 2015 Accepted: 22 February 2016 Published online: 08 April 2016

\section{References}

1. Chua, LO: Memristor - the missing circuit element. IEEE Trans. Circuit Theory 18(5), 507-511 (1971)

2. Strukov, DB, Snider, GS, Stewart, DR, Williams, RS: The Missing Memristor Found. Nature 453, 80-83 (2008) 
3. Bayat, FM, Shouraki, SB: Programming of memristor crossbars by using genetic algorithm. Proc. Comput. Sci. 3, 232-237 (2011)

4. Itoh, M, Chua, LO: Memristor oscillators. Int. J. Bifurc. Chaos 18, 3183-3206 (2008)

5. Pakkiyappan, R, Sivasamy, R, Li, XD: Synchronization of identical and nonidentical memristor-based chaotic systems via active back stepping control technique. Circuits Syst. Signal Process. 34(3), 763-778 (2015)

6. Wu, HG, Chen, SY, Bao, BC: Impulsive synchronization and initial value effect for a memristor-based chaotic system. Acta Phys. Sin. 64(3), 030501 (2015)

7. Wang, X, Li, CD, Huang, TW, Duan, SK: Predicting chaos in memristive oscillators via harmonic balance method. Chaos 22(4), $043119(2012)$

8. Bao, HB, Cao, JD: Projective synchronization of fractional-order memristor-based neural networks. Neural Netw. 63, 1-9 (2015)

9. Wen, SP, Zeng, ZG, Huang, TW: Adaptive synchronization of memristor-based Chua's circuits. Phys. Lett. A 376, $2275-2780(2012)$

10. Huang, JJ, Li, CD, He, X: Stabilization of a memristor-based chaotic system by intermittent and fuzzy processing. Int. J. Control. Autom. Syst. 11(3), 643-647 (2013)

11. Amato, F, Ariola, M, Abdallah, CT: Finite-time control for uncertain linear systems with disturbance inputs. In: IEEE Proceedings of the 1999 American Control Conference, vol. 3, pp. 1776-1780 (1999)

12. Amato, F, Arioila, M, Dorato, P: Finite-time control of linear systems subject to parametric uncertainties and disturbances. Automatica 37(9), 1459-1463 (2001)

13. Amato, F, Arioila, M, Cosentino, C: Finite-time stability of linear time-varying systems: analysis and controller design IEEE Trans. Autom. Control 55(4), 1003-1008 (2009)

14. Sanjay, PB, Dennis, SB: Finite time of continuous autonomous systems. SIAM J. Control Optim. 3(3), 751-766 (2000)

15. Liu, YG: Global finite time stabilization via time varying feedback uncertain nonlinear systems. SIAM J. Control Optim. 52(3), 1886-1913 (2014)

16. Li, X, Bohner, M, Wang, CK: Impulsive differential equations: periodic solutions and applications. Automatica 52, 173-178 (2015)

17. Li, X, Song, S: Impulsive control for existence, uniqueness and global stability of periodic solutions of recurrent neural networks with discrete and continuously distributed delays. IEEE Trans. Neural Netw. 24, 868-877 (2013)

18. Wen, SP, Huang, TW, Yu, XH, Chen, MZQ, Zeng, ZG: Aperiodic sampled-data sliding-mode control of fuzzy systems with communication delays via the event-triggered method. IEEE Trans. Fuzzy Syst. (2015). doi:10.1109/TFUZZ.2015.2501412

19. Wen, SP, Yu, XH, Zeng, ZG, Wang, JJ: Event-triggering load frequency control for multi-area power systems with communication delay. IEEE Trans. Ind. Electron. 63(2), 1308-1317 (2015)

20. Song, QK, Huang, TW: Stabilization and synchronization of chaotic systems with mixed time-varying delays via intermittent control with non-fixed both control period and control width. Neurocomputing 154, 61-69 (2015)

21. Song, QK, Zhao, ZJ: Stability criterion of complex-valued neural networks with both leakage delay and time-varying delays on time scales. Neurocomputing 171, 179-184 (2016)

22. Cao, JD, Song, QK: Stability in Cohen-Grossberg-type bidirectional associative memory neural networks with time-varying delays. Nonlinearity 19(7), 1601 (2006)

\section{Submit your manuscript to a SpringerOpen ${ }^{\circ}$ journal and benefit from:}

- Convenient online submission

Rigorous peer review

- Immediate publication on acceptance

- Open access: articles freely available online

- High visibility within the field

- Retaining the copyright to your article 